\title{
Association between neutrophil-to-lymphocyte ratio, platelet-to-lymphocyte ratio, and diabetic retinopathy among diabetic patients without a related family history
}

Jin-Rui Wang ${ }^{1 \dagger}$, Zhongli Chen ${ }^{2 \dagger}$, Ke Yang ${ }^{2}$, Hui-Jun Yang ${ }^{1}$, Wen-Yu Tao ${ }^{1}$, Yi-Ping Li ${ }^{1}$, Ze-Jia Jiang ${ }^{3}$, Chao-Fang Bai ${ }^{1}$, Yue-Chuan Yin ${ }^{1}$, Jian-Mei Duan ${ }^{1}$, Yuan-Yuan Zhou ${ }^{1}$, Xin-Qian Geng ${ }^{1}$ and Ying Yang ${ }^{1 *}$ (D)

\begin{abstract}
Background: Diabetic retinopathy (DR) is a specific neurovascular complication of diabetes mellitus (DM). Clinically, family history is a widely recognized risk factor for DR, assisting diagnosis and risk strata. However, among a great amount of DR patients without hereditary history like hypertension and diabetes, direct and simple risk factors to assist clinical decisions are still required. Herein, we intend to investigate the associated risk factors for these DR patients based on systemic inflammatory response indexes, neutrophil-to-lymphocyte ratio (NLR) and platelet-tolymphocyte ratio (PLR).
\end{abstract}

Methods: We consecutively enrolled 1030 patients with a definite diagnosis of type 2 diabetes mellitus (T2DM) from the endocrinology department of the Second hospital of People in Yun Nan. Based on funduscopy and family history checking, we excluded patients with a family history of hypertension and diabetes and finally enrolled 264 patients with DR and 206 patients with non-diabetic retinopathy (NDR). Through correlation analysis, univariate and multivariate regression, we further explore the association between NLR, PLR, and DR. On top of that, we investigate the effect of NLR and PLR on risk reclassification of DR.

Results: Compared with NDR patients, NLR and PLR levels are significantly higher among DR patients (NLR: $2.36 \pm 1.16$ in DR group versus $1.97 \pm 1.06$ in NDR group, $p<0.001 ; P L R: 11.62 \pm 4.55$ in DR group versus $10.56 \pm 4.45$ in NDR group, $p=0.012$ ). According to univariate analysis, NLR and PLR add risks to DR. After fully adjusting co-founders, $\mathrm{NLR}$, as both continuous and categorical variate, remains an independent risk factor for DR (OR $(95 \% \mathrm{Cl}): 1.37(1.06$, 1.78) $P=0.018)$. And though PLR was not independently associated with $\mathrm{DR}$ as a continuous variable $(\mathrm{OR}(95 \% \mathrm{Cl}) 1.05$ $(0.99,1.11) p=0.135)$, the highest quantile of PLR add two-fold increased risk (OR $(95 \% \mathrm{Cl}) 2.20(1.05,4.59) p=0.037)$ in the fully adjusted model for DR. In addition, addition of PLR and NLR to the established factor hemoglobin (Hb) improved the discriminability of the model and assisted the reclassification of DR. After combining PLR and NLR the

\footnotetext{
*Correspondence: yangying2072@126.com

†'Jin-Rui Wang and Zhongli Chen contributed equally to this study ${ }^{1}$ Department of Endocrinology and Metabolism, The Second People's Hospital of Yunnan Province, Fourth Affiliated Hospital of Kunming Medical University, Kunming 650021, China

Full list of author information is available at the end of the article
}

(c) The Author(s) 2020. This article is licensed under a Creative Commons Attribution 4.0 International License, which permits use, sharing, adaptation, distribution and reproduction in any medium or format, as long as you give appropriate credit to the original author(s) and the source, provide a link to the Creative Commons licence, and indicate if changes were made. The images or other third party material in this article are included in the article's Creative Commons licence, unless indicated otherwise in a credit line to the material. If material is not included in the article's Creative Commons licence and your intended use is not permitted by statutory regulation or exceeds the permitted use, you will need to obtain permission directly from the copyright holder. To view a copy of this licence, visit http://creativeco mmons.org/licenses/by/4.0/. The Creative Commons Public Domain Dedication waiver (http://creativecommons.org/publicdomain/ zero/1.0/) applies to the data made available in this article, unless otherwise stated in a credit line to the data. 
Area under curve (AUC) of $\mathrm{Hb}$ based model raised from 0.76 to 0.78 , with a category-free net reclassification improvement (NRI) of $0.532(p<0.001)$ and integrated discrimination improvement (IDI) of $0.029(p<0.001)$.

Conclusions: Systemic inflammatory response indexes NLR and PLR were associated with the presence of DR among patients without associated family history and contributed to improvements in reclassification of DR in addition to $\mathrm{Hb}$.

Keywords: Type 2 diabetes mellitus, Diabetic retinopathy, Family history, Neutrophil-to-lymphocyte ratio, Platelet-tolymphocyte ratio, Hemoglobin

\section{Background}

Diabetic retinopathy (DR), as a specific neurovascular complication of diabetes mellitus(DM),become a major reason for blinding people aged 20-74 [1]. The pathogenesis of DR was complicated, with diverse factors involvement. According to the major researches, commonly acknowledged risk factors include family history, hyperglycemia, hypertension, and hyperlipidemia, long-duration of diabetes, diabetic nephropathy, blood glucose fluctuation, obesity and gestation [2-5]. Particularly, inheritable and unchangeable factors such as genetic polymorphism were closely associated with the incidence and progress of DR [5]. Due to the high costs and low cooperation rate among patients, family history was regarded as a direct or indirect interpretation of inheritable risk factor which helps clinicians to evaluate the risk for DR. It is reported that presence of family history, diabetes, and hypertension, always indicated lower age of the patients which is associated to rather longer duration of diabetes for these patients [6, 7]. In addition, researches revealed a family history of diabetes and hypertension also contributed to a higher frequency of DR $[8,9]$. However, in clinical practice, despite the complicated inheritable factors, a large number of diabetic patients without obvious family history also take up a major part of DR patients. Exploring risk factors for these people is quite important for clinical decisions.

Recently, increasing studies reported that routine blood examination could provide rich and effective information to assist risk stratification of disease [10, 11].The promising index neutrophil-to-lymphocyte ratio (NLR) and platelet-to-lymphocyte ratio (PLR) conveyed a systematic inflammatory response in our body and has been evidenced as predictive and prognostic factors for DM and the related complications [12-15]. Moreover, Hemoglobin $(\mathrm{Hb})$ was also a well-established biomarker for DR $[16,17]$. However, the association between these factors and DR among patients without diabetes or hypertension family history remains unclear. Herein, our study aimed at clarifying the relationship between NLR, PLR, and DR in patients without inheritable risk factors, and further detect the contribution of NLR and PLR to disease reclassification when combining the traditional factor $\mathrm{Hb}$.

\section{Methods}

\section{Participants and study design}

We consecutively enrolled a total of 1030 type 2 diabetic patients undergoing funduscopy from the endocrinology department of the Second hospital of People in Yun Nan. Diagnosis of type 2 diabetes was made according to the 1999 World Health Organization criteria [18]. The diagnosis of diabetic was based on the 2002 International Clinical Classification Standard [19]. And the non-diabetic retinopathy group (NDR) was defined as patients who had been clearly diagnosed with type 2 diabetes and had no diabetic retinopathy on fundus. Besides patients with a family history of hypertension and diabetes, patients with severe systemic disease, glaucoma, trauma, non-diabetic retinopathy, pregnancy, malignant tumors, severe cardiovascular and cerebrovascular diseases, liver and kidney dysfunction, blood disease, recent surgery, infection or other severe stress condition were also excluded. Finally, we successfully enrolled 264 patients with DR and 206 patients without DR (the NDR group).

\section{Clinical information and biochemical examination}

All participants received routine examination and blood examination and were asked in detail about their disease history, medical history, and personal history. After collecting patients fasting peripheral blood, blood routine test and white blood cell classification were performed. Specifically, the blood routine and blood cell count were detected using a fully automated blood analyzer from Xisen Meikang (XE2100, Japan); nucleic acid fluorescent staining, laser counting and flow cytometry were used to determine white blood cells and white blood cell classification; colorimetric method was used to determine hemoglobin concentration; and double sheath flow impedance method was applied to determine platelet count and red blood cell count. Neutrophil-to-lymphocyte ratio (NLR), platelet-to-lymphocyte ratio (PLR) and monocyte-to-lymphocyte ratio (MLR) were calculated for all blood samples. All biochemical analyses of the same samples were performed in our hospital, including the analyses of fasting blood glucose (FPG), glycosylated hemoglobin A1c (HbA1c), triglyceride (TG), total cholesterol (TC), low-density lipoprotein 
cholesterol (LDL-C), high-density lipoprotein cholesterol (HDL-C), serum creatinine (Scr), and blood urea nitrogen (BUN). Among them, the glucose determination kit (glycokinase method) was used to determine fasting blood glucose; the HbA1c determination kit (enzymatic method) was used to determine HbA1c in plasma; the creatinine detection kit (enzymatic method) was used to determine serum creatinine; the urea nitrogen detection kit (Enzymatic method) were used for determination of serum urea nitrogen level. Total cholesterol kit (liquid) (oxidase method), triglyceride kit (liquid) (glycerol phosphate oxidase method), lipoprotein cholesterol detection kit (direct method), and low-density lipoprotein cholesterol detection kit (direct method) were used for measurements of total cholesterol, triglyceride, high-density lipoprotein cholesterol and low-density lipoprotein cholesterol in serum, respectively.

\section{Statistics analysis}

Distribution normality was initially tested through the Kolmogorov-Smirnov test. Continuous data are showed by the mean \pm standard deviation (SD) and were compared by independent Student $t$ test or oneway analysis of variance test. While variables without normality were expressed by median plus IQR. The Chi squared test or Fisher's exact test was used to compare categorical variables. Due to the order of magnitude of PLR, a scaling factor of 10 was included to explain unit conversion. The correlation was assessed by the Spearman correlation test. NLR, PLR were analyzed as continuous variates and also respectively divided into quartiles, with the first quartile representing the lowest levels and the fourth quartile the highest. Univariate logistic regression models were used to examine possible factors associated with DR. Multivariable logistic regression analysis was conducted to assess the independent association between NLR, PLR and DR. Variables with known clinical risk, significance at the $5 \%$ level from the univariate analyses were included as co-founders in for an adjustment. After adjusting for age, gender, diabetes duration, hypertension, BMI, Scr, white blood cell (WBC), Hb, BUN, TG, FPG and HBA1c, odds ratios with 95\% confidence intervals were reported. In logistics regression, an Akaike information criterion (AIC) based stepwise variable selection method was used to acquire the optimal logistic regression model.

All statistical analyses were performed by SPSS software (version 22.0; SPSS, Inc., Chicago, IL, USA). P-values less than $0.05(\mathrm{P}<0.05)$ were considered significant and the significance was two-tailed.

\section{Results}

Baseline

As shown in Table 1, gender, presence of hypertension, smoking, drinking, diabetes duration, FPG, WBC, Hb, BUN, Scr and TG are significantly different between DR and NDR group $(\mathrm{P}<0.05)$. Specifically, compared with the NDR group, DR patients have longer diabetes duration, higher levels of FPG, WBC, BUN, Scr and TG, a higher proportion of female and hypertension presence. However, levels of $\mathrm{Hb}$ were lower in DR patients. In addition, no difference was observed

Table 1 Baseline characteristics of the DR and NDR groups

\begin{tabular}{|c|c|c|c|}
\hline Variable & $\begin{array}{l}\text { DR group } \\
N=264\end{array}$ & $\begin{array}{l}\text { NDR group } \\
\mathrm{N}=206\end{array}$ & $P$ \\
\hline $\begin{array}{l}\text { Gender (male/ } \\
\text { female) }\end{array}$ & 131/133 & $146 / 60$ & $0.000^{* *}$ \\
\hline $\begin{array}{l}\text { Hypertension (no/ } \\
\text { yes) }\end{array}$ & $95 / 169$ & $108 / 98$ & $0.000^{* *}$ \\
\hline Smoking (no/yes) & $168 / 96$ & $107 / 99$ & $0.007^{*}$ \\
\hline Drinking (no/yes) & $180 / 84$ & $119 / 87$ & $0.013^{*}$ \\
\hline $\begin{array}{l}\text { Sulfonylureas (no/ } \\
\text { yes) }\end{array}$ & $147 / 117$ & $110 / 96$ & 0.344 \\
\hline Biguanides (no/yes) & $129 / 135$ & $95 / 110$ & 0.327 \\
\hline Insulin (no/yes) & $127 / 137$ & $108 / 98$ & 0.201 \\
\hline Age (years) & $56.48 \pm 9.86$ & $55.44 \pm 11.27$ & 0.295 \\
\hline NLR & $2.36 \pm 1.16$ & $1.97 \pm 1.06$ & $0.000^{* *}$ \\
\hline $\operatorname{MLR}\left({ }^{*} 10\right)$ & $2.28 \pm 1.03$ & $2.34 \pm 1.28$ & 0.628 \\
\hline $\operatorname{PLR}\left({ }^{*} 0.1\right)$ & $11.62 \pm 4.55$ & $10.56 \pm 4.45$ & $0.012^{*}$ \\
\hline Neutrophils $\left({ }^{*} 10^{9} / \mathrm{L}\right)$ & $4.25 \pm 1.31$ & $3.72 \pm 1.22$ & $0.000^{* *}$ \\
\hline $\begin{array}{l}\text { Lymphocytes } \\
\qquad\left({ }^{*} 10^{9} / \mathrm{L}\right)\end{array}$ & $1.98 \pm 0.64$ & $2.11 \pm 0.67$ & $0.031^{*}$ \\
\hline Monocytes $\left({ }^{*} 10^{9} / \mathrm{L}\right)$ & $0.42 \pm 0.15$ & $0.44 \pm 0.15$ & 0.097 \\
\hline Platelets $\left({ }^{*} 10^{9} / \mathrm{L}\right)$ & $213.84 \pm 69.19$ & $203.90 \pm 54.66$ & 0.092 \\
\hline $\begin{array}{l}\text { Diabetes course } \\
\text { (month) }\end{array}$ & $122.86 \pm 87.38$ & $66.16 \pm 66.25$ & $0.000^{* *}$ \\
\hline $\mathrm{BMI}\left(\mathrm{kg} / \mathrm{m}^{2}\right)$ & $24.12 \pm 3.25$ & $24.62 \pm 3.14$ & 0.090 \\
\hline FPG (mmol/L) & $9.60 \pm 3.79$ & $8.80 \pm 3.27$ & $0.019 *$ \\
\hline $\mathrm{HbA1c}(\%)$ & $9.96 \pm 2.45$ & $9.68 \pm 2.66$ & 0.246 \\
\hline WBC $\left({ }^{*} 10^{9} / \mathrm{L}\right)$ & $6.83 \pm 1.65$ & $6.45 \pm 1.50$ & $0.010^{*}$ \\
\hline $\mathrm{Hb}(\mathrm{g} / \mathrm{L})$ & $136.25 \pm 19.33$ & $153.20 \pm 15.70$ & $0.000^{* *}$ \\
\hline BUN (mmol/L) & $7.07 \pm 7.82$ & $5.32 \pm 1.64$ & $0.000^{* *}$ \\
\hline $\mathrm{Scr}(\mathrm{mmol} / \mathrm{L})$ & $84.21 \pm 46.63$ & $69.14 \pm 17.87$ & $0.000^{* *}$ \\
\hline $\mathrm{TG}(\mathrm{mg} / \mathrm{dL})$ & $3.02 \pm 2.37$ & $2.29 \pm 1.99$ & $0.000^{* *}$ \\
\hline $\mathrm{TC}(\mathrm{mg} / \mathrm{dL})$ & $4.73 \pm 2.09$ & $4.79 \pm 1.13$ & 0.694 \\
\hline $\mathrm{HDL}-\mathrm{C}(\mathrm{mg} / \mathrm{dL})$ & $1.16 \pm 0.33$ & $1.12 \pm 0.57$ & 0.434 \\
\hline $\mathrm{LDL}-\mathrm{C}(\mathrm{mg} / \mathrm{dL})$ & $3.11 \pm 0.98$ & $2.97 \pm 0.88$ & 0.110 \\
\hline
\end{tabular}

$D R$ diabetic retinopathy, NDR non-diabetic retinopathy, NLR neutrophilto-lymphocyte ratio, $M L R$ monocyte-to-lymphocyte ratio, $P L R$ platelet-tolymphocyte ratio, BMI body mass index, FPG fasting plasma glucose, $\mathrm{HbA1C}$ glycated hemoglobin A1c, WBC white blood cell, $H b$ hemoglobin, BUN blood urea nitrogen, $S c r$ serum creatinine, $T G$ triglycerides, $T C$ total cholesterol, $H D L-C$ high density lipoprotein cholesterol, $L D L-C$ low density lipoprotein cholesterol ${ }^{*} P<0.05,{ }^{*} P<0.01$ 
in age, BMI, HBA1c, TC, LDL-C, HDL-C levels as well as the treatment of diabetes. According to Fig. 1, levels of NLR $(2.36 \pm 1.16$ in DR group versus $1.97 \pm 1.06$ in NDR group, $\mathrm{p}<0.001)$ and $\operatorname{PLR}(11.62 \pm 4.55$ in DR group versus10.56 \pm 4.45 in NDR group, $\mathrm{p}=0.012$ ) were significantly higher in DR group.

\section{Correlation analysis of NLR, PLR and $\mathrm{Hb}$ and major clinical} factors

As shown in Table 2, according to correlation analysis, NLR positively correlated with MLR, PLR, diabetes duration, BUN and Scr, and negatively correlated with $\mathrm{Hb}$, but displayed insignificant correlation with age, gender, hypertension, BMI, FPG, and HbA1c. Meanwhile, PLR correlated positively with NLR, MLR and diabetes duration, and negatively with BMI and Hb. In addition,
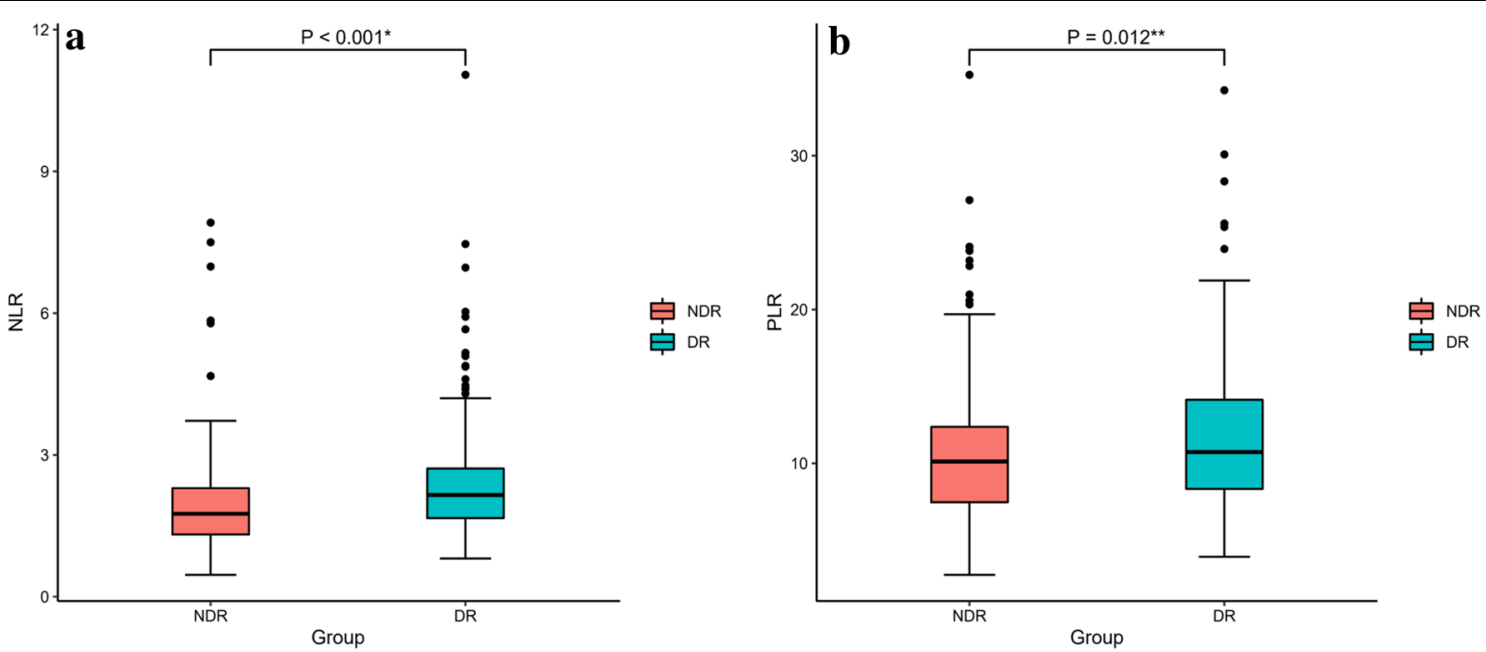

Fig. 1 Comparison of NLR and PLR in different group a Boxplot showed that DR patients had higher NLR than the NDR patients $\left(p<0.001^{* *}\right)$. b Boxplot showed that DR patients had higher PLR than the NDR patients $(p=0.012)$

Table 2 Correlation analysis of NLR, PLR and $\mathrm{Hb}$ and major clinical factors

\begin{tabular}{|c|c|c|c|c|c|c|}
\hline \multirow[t]{2}{*}{ Variable } & \multicolumn{2}{|l|}{ NLR } & \multicolumn{2}{|c|}{$\operatorname{PLR}(* 0.1)$} & \multicolumn{2}{|l|}{$\mathrm{Hb}(\mathrm{g} / \mathrm{L})$} \\
\hline & $\mathbf{R}$ & $P$ & $\mathbf{R}$ & $P$ & $\mathbf{R}$ & $P$ \\
\hline Gender (male/female) & -0.061 & 0.190 & 0.080 & 0.082 & -0.465 & $0.000^{* *}$ \\
\hline Hypertension (no/yes) & 0.053 & 0.247 & -0.008 & 0.855 & -0.130 & $0.005^{* *}$ \\
\hline Age (years) & 0.055 & 0.238 & 0.029 & 0.534 & -0.150 & $0.001^{* *}$ \\
\hline NLR & 1 & 1 & 0.520 & $0.000^{* *}$ & -0.115 & $0.012^{*}$ \\
\hline $\operatorname{MLR}\left({ }^{*} 10\right)$ & 0.609 & $0.000^{* *}$ & 0.446 & $0.000^{* *}$ & 0.004 & 0.934 \\
\hline $\operatorname{PLR}\left({ }^{*} 0.1\right)$ & 0.520 & $0.000^{* *}$ & 1 & 1 & -0.289 & $0.000^{* *}$ \\
\hline Diabetes course (month) & 0.125 & $0.007^{* *}$ & 0.093 & $0.044^{*}$ & -0.266 & $0.000^{* *}$ \\
\hline BMI $\left(\mathrm{kg} / \mathrm{m}^{2}\right)$ & -0.021 & 0.651 & -0.117 & $0.011^{*}$ & 0.194 & $0.000^{* *}$ \\
\hline FPG (mmol/L) & 0.015 & 0.755 & -0.063 & 0.184 & 0.080 & 0.091 \\
\hline HbA1c (\%) & -0.001 & 0.980 & -0.072 & 0.129 & 0.076 & 0.110 \\
\hline $\mathrm{Hb}(\mathrm{g} / \mathrm{L})$ & -0.115 & $0.012^{*}$ & -0.289 & $0.000^{* *}$ & 1 & 1 \\
\hline BUN (mmol/L) & 0.100 & $0.033^{*}$ & -0.042 & 0.371 & -0.220 & $0.000^{* *}$ \\
\hline $\mathrm{Scr}(\mathrm{mmol} / \mathrm{L})$ & 0.173 & $0.000^{* *}$ & 0.079 & 0.092 & -0.065 & 0.168 \\
\hline Sulfonylureas (no/yes) & -0.078 & 0.090 & 0.049 & 0.291 & -0.016 & 0.725 \\
\hline Biguanides (no/yes) & 0.033 & 0.476 & -0.058 & 0.209 & 0.072 & 0.120 \\
\hline Insulin (no/yes) & 0.081 & 0.081 & -0.032 & 0.491 & 0.009 & 0.839 \\
\hline
\end{tabular}

$N L R$ neutrophil-to-lymphocyte ratio, MLR monocyte-to-lymphocyte ratio, $P L R$ platelet-to-lymphocyte ratio, $B M I$ body mass index, FPG fasting plasma glucose, $H b A 1 C$ glycated hemoglobin A1c, $H b$ hemoglobin, $B U N$ blood urea nitrogen, $S c r$ serum creatinine, $R$ correlation coefficient

${ }^{*} P<0.05,{ }^{* *} P<0.01$ 
$\mathrm{Hb}$ correlated positively with BMI and negatively correlated with gender, hypertension, age, NLR, PLR, diabetes course, and BUN.

\section{Univariate analysis}

As shown in Table 3, our univariate regression analysis revealed that gender of female, presence of hypertension, long course of diabetes, higher levels of FPG, WBC, NLR,

Table 3 Univariate analysis

\begin{tabular}{lllll}
\hline Variable & OR & $\begin{array}{l}\mathbf{9 5 \% C l} \\
\text { lower }\end{array}$ & $\begin{array}{l}\mathbf{9 5 \% C l} \\
\text { upper }\end{array}$ & $\boldsymbol{P}$ \\
\hline Gender (Male/Female) & 2.47 & 1.68 & 3.63 & $0.000^{* *}$ \\
Hypertension (no/yes) & 1.96 & 1.35 & 2.84 & $0.000^{* *}$ \\
Age (years) & 1.01 & 0.99 & 1.03 & 0.295 \\
NLR & 1.46 & 1.19 & 1.79 & $0.000^{* *}$ \\
MLR (*10) & 0.96 & 0.82 & 1.13 & 0.619 \\
PLR (*0.1) & 1.06 & 1.01 & 1.10 & $0.013^{*}$ \\
Diabetes course (month) & 1.01 & 1.01 & 1.01 & $0.000^{* *}$ \\
BMI (Kg/m2) & 0.95 & 0.90 & 1.01 & 0.090 \\
FPG (mmol/L) & 1.07 & 1.01 & 1.13 & $0.020^{*}$ \\
HbA1c (\%) & 1.05 & 0.97 & 1.13 & 0.239 \\
WBC $\left.{ }^{*} 10^{9} / \mathrm{L}\right)$ & 1.17 & 1.04 & 1.31 & $0.010^{*}$ \\
Hb (g/L) & 0.95 & 0.93 & 0.96 & $0.000^{* *}$ \\
BUN (mmol/L) & 1.42 & 1.26 & 1.59 & $0.000^{* *}$ \\
Scr (mmol/L) & 1.02 & 1.01 & 1.02 & $0.000^{* *}$ \\
TG (mg/dL) & 1.18 & 1.07 & 1.31 & $0.001^{* *}$ \\
TC (mg/dL) & 0.98 & 0.88 & 1.09 & 0.693 \\
HDL-C (mg/dL) & 1.21 & 0.76 & 1.93 & 0.412 \\
LDL-C (mg/dL) & 1.17 & 0.12 & 1.43 & 0.110 \\
\hline
\end{tabular}

NLR neutrophil-to-lymphocyte ratio, $M L R$ monocyte-to-lymphocyte ratio, $P L R$ platelet-to-lymphocyte ratio, $B M /$ body mass index, $F P G$ fasting plasma glucose $H b A 1 c$ glycated hemoglobin A1c, WBC white blood cell, $H b$ Hemoglobin, BUN blood urea nitrogen,Scr serum creatinine, TG triglycerides, TC total cholesterol, HDL-C high density lipoprotein cholesterol, LDL-C low density lipoprotein cholesterol, $O R$ odds ratios, $C l$ confidence intervals

${ }^{*} P<0.05,{ }^{*} P<0.01$
PLR BUN, Scr and TG adds risk to presence of DR, while higher counts of $\mathrm{Hb}$ was related to lower risk of DR. However, no association was found between age, BMI, HbA1c, TC, HDL-C, LDL-C, MLR and presence of DR.

\section{Associations between NLR, PLR, and DR $N L R$ and $D R$}

By conducting multivariate analysis, we found that NLR was associated with DR independent of other known factors. With a unit increase of NLR, the risk for DR would raise $37 \%$. Furthermore, when treated as a category variate divided according to its quantile, the association of NLR and DR still exists. As Table 4 demonstrated, from the crude model to simple or complex model, there was a 2.8 fold increased risk for DR in the highest quantile of NLR (OR, 95\%CI $2.80(1.32,5.95) \mathrm{p}=0.007)$ (Table 4).

\section{PLR and DR}

We also included PLR in multivariate logistics regression by adjusting other co-founders and observed that PLR was not an independent risk factor for DR as a continuous variable, but the ranked level of the index assists the risk stratification. Specifically, after full adjustment, the highest quantile of PLR held add 2.2 times of risk to the presence of DR compared with the first quantile(OR(95\%CI)2.20 (1.05, 4.59), $\mathrm{P}=0.037)$ (Table 5).

\section{NLR and PLR for reclassification of DR $H b$ and $D R$}

Particularly, we also assess the performance of the previous factor-Hb in our diabetic patients' group. By applying crude and different multivariate models, we confirmed that no matter treated as a continuous variable or categorical variable, $\mathrm{Hb}$ was stably related to lower risk of $\mathrm{DR}$ with the higher quartile displaying lower risk probability (Table 6).

\section{Table 4 Independent association between NLR and DR}

\begin{tabular}{llll}
\hline Exposure & Model & Model I & Model II \\
\hline NLR group & OR $(95 \% \mathrm{Cl}) P$ & OR $(95 \% \mathrm{Cl}) P$ & OR $(95 \% \mathrm{Cl}) P$ \\
ContinuousNLR & $1.46(1.19,1.78) 0.000^{* *}$ & $1.46(1.18,1.81) 0.000^{* *}$ & $1.37(1.06,1.78) 0.018^{*}$ \\
$0.46-1.49$ & 1 & 1 & 1 \\
$1.50-1.95$ & $1.89(1.12,3.17) 0.016^{*}$ & $2.11(1.19,3.73) 0.011^{*}$ & $1.23(0.62,2.44) 0.556$ \\
$1.95-2.54$ & $2.25(1.33,3.79) 0.002^{* *}$ & $2.51(1.42,4.46) 0.002^{* *}$ & $1.77(0.88,3.55) 0.110$ \\
$2.54-11.05$ & $3.44(2.01,5.89) 0.000^{* *}$ & $3.61(1.99,6.55) 0.000^{* *}$ & $2.80(1.32,5.95) 0.007^{* *}$ \\
P for trend & $0.000^{* *}$ & $0.000^{* *}$ & $0.004^{* *}$ \\
\hline
\end{tabular}

Model: crude model; Model I: adjusting for age, gender, diabetes duration; Model II: adjusting for age, gender, diabetes duration, hypertension, BMI, Scr, WBC, Hb, BUN, TG, FPG and HbA1c

$N L R$ neutrophil-to-lymphocyte ratio, $O R$ odds ratios, $C I$ confidence intervals, $B M I$ body mass index, Scr serum creatinine, WBC white blood cell, $H b$ hemoglobin, $B U N$ blood urea nitrogen, TG triglycerides, FPG fasting plasma glucose, HbA1c glycated hemoglobin A1c

${ }^{*} P<0.05,{ }^{*} P<0.01$ 
A combination of PLR, NLR, and $\mathrm{Hb}$ for predicting DR In order to evaluate the prognostic value of NLR and PLR for improving risk stratification of DR, we performed a receiver-operating characteristic (ROC) analyses to calculate the area under the curve (AUC) of each factor and assess the performance of a combination of these factors (Table 7). Though NLR and PLR alone didn't perform better than $\mathrm{Hb}$, a combination of NLR, PLR, and $\mathrm{Hb}$ indeed result in a model with increased predictive performance (area under the ROC curve 0.78 (95\%CI 0.74-0.82) versus. 0.76 (95\%CI 0.72-0.81)) (Fig. 2). Furthermore, the addition of NLR, PLR significantly improved the risk reclassification over using $\mathrm{Hb}$ alone, with a considerable category-free net reclassification improvement(NRI) and a meaningful integrated discrimination improvement (IDI) for DR among diabetic patients without a

Table 5 Independent association between PLR and DR

\begin{tabular}{llll}
\hline Exposure & Model & Model I & Model II \\
\hline PLRgroup & OR $(95 \% \mathrm{Cl}) P$ & OR $(95 \% \mathrm{Cl}) P$ & OR $(95 \% \mathrm{Cl})$ \\
Continuous PLR & $1.06(1.01,1.10) 0.013^{*}$ & $1.04(0.99,1.09) 0.109$ & $1.05(0.99,1.11) 0.135$ \\
$2.75-7.98$ & 1 & 1 & 1 \\
$7.99-10.34$ & $1.65(0.98,2.76) 0.058$ & $1.86(1.06,3.27) 0.031^{*}$ & $1.37(0.70,2.71) 0.360$ \\
$10.36-13.40$ & $1.17(0.70,1.94) 0.558$ & $1.34(0.77,2.34) 0.307$ & $0.95(0.47,1.92) 0.891$ \\
$13.49-35.26$ & $2.51(1.47,4.27) 0.000^{* *}$ & $2.15(1.20,3.85) 0.001^{* *}$ & $2.20(1.05,4.59) 0.037^{*}$ \\
P for trend & $0.003^{* *}$ & $0.033^{*}$ & 0.073
\end{tabular}

Model: crude model; Model I: adjusting for age, gender, diabetes duration; Model Il: adjusting for age, gender, diabetes duration, hypertension, BMI, Scr, WBC, Hb, BUN, TG, FPG and HbA1c

$P L R$ platelet-to-lymphocyte ratio, $O R$ odds ratios, $C I$ confidence intervals, $B M I$ body mass index, Scr serum creatinine, WBC white blood cell, $H b$ hemoglobin, $B U N$ blood urea nitrogen, TG triglycerides, FPG fasting plasma glucose, HbA1c glycated hemoglobin A1c

${ }^{*} P<0.05,{ }^{* *} P<0.01$

Table 6 Independent association between $\mathrm{Hb}$ and DR

\begin{tabular}{llll}
\hline Exposure & Model & Model I & Model II \\
\hline Hb group & OR $(95 \% \mathrm{Cl}) P$ & OR $(95 \% \mathrm{Cl}) P$ & OR $(95 \% \mathrm{Cl}) P$ \\
Continuous $\mathrm{Hb}$ & $0.95(0.93,0.96) 0.000^{* *}$ & $0.95(0.94,0.97) 0.000^{* *}$ & $0.96(0.94,0.97) 0.000^{* *}$ \\
$70-132(\mathrm{~g} / \mathrm{L})$ & 1 & 1 & 1 \\
$133-143(\mathrm{~g} / \mathrm{L})$ & $0.32(0.17,0.61) 0.000^{* *}$ & $0.38(0.19,0.73) 0.004^{* *}$ & $0.88(0.44,1.74) 0.707$ \\
$144-157(\mathrm{~g} / \mathrm{L})$ & $1.61(0.95,2.71) 0.076$ & $2.02(1.12,3.62) 0.019^{*}$ & $0.27(0.12,0.60) 0.001^{* *}$ \\
$158-195(\mathrm{~g} / \mathrm{L})$ & $0.07(0.04,0.13) 0.000^{* *}$ & $0.09(0.05,0.20) 0.000^{* *}$ & $0.95(0.93,0.97) 0.000^{* *}$ \\
P for trend & $0.000^{* *}$ & $0.000^{* *}$ & $0.000^{* *}$
\end{tabular}

Model: Univariate model; Model I: adjusting for age, gender, and diabetes course; Model II: adjusting for age, gender, diabetes course, hypertension, BMI, Scr, WBC, NLR, PLR, BUN, TG, FPG, HbA1C

$H b$ hemoglobin, $O R$ odds ratios, $C l$ confidence intervals, $B M I$ body mass index, Scr serum creatinine, WBC white blood cell, NLR neutrophil-to-lymphocyte ratio, $P L R$ platelet-to-lymphocyte ratio, BUN blood urea nitrogen, TG triglycerides, FPG fasting plasma glucose, $H b A 1 c$ glycated hemoglobin A1c

${ }^{*} P<0.05,{ }^{* *} P<0.01$

Table 7 A combination of PLR, NLR and Hb for predicting DR

\begin{tabular}{|c|c|c|c|c|c|c|}
\hline Marker & AUC & $95 \% \mathrm{Cl}$ lower & $95 \% \mathrm{Cl}$ upper & Cut off & Specificity & Sensitivity \\
\hline NLR & 0.64 & 0.58 & 0.69 & 1.84 & 0.56 & 0.64 \\
\hline PLR & 0.58 & 0.53 & 0.63 & 128.11 & 0.79 & 0.35 \\
\hline $\mathrm{Hb}$ & 0.76 & 0.72 & 0.81 & 146.50 & 0.68 & 0.72 \\
\hline $\mathrm{NLR}+\mathrm{PLR}+\mathrm{Hb}$ & 0.78 & 0.74 & 0.82 & - & 0.68 & 0.75 \\
\hline
\end{tabular}

Based on model: logit $(D R)=8.74874-0.06060 * \mathrm{Hb}+0.49400 * \mathrm{NLR}-0.00682 * \mathrm{PLR}$

AUC area under curve, $C /$ confidence intervals, NLR neutrophil-to-lymphocyte ratio, $P L R$ platelet-to-lymphocyte ratio, $H b$ hemoglobin, $N R I$ net reclassification index, IDI integrated discrimination improvement 


\section{ROC curves for DR}

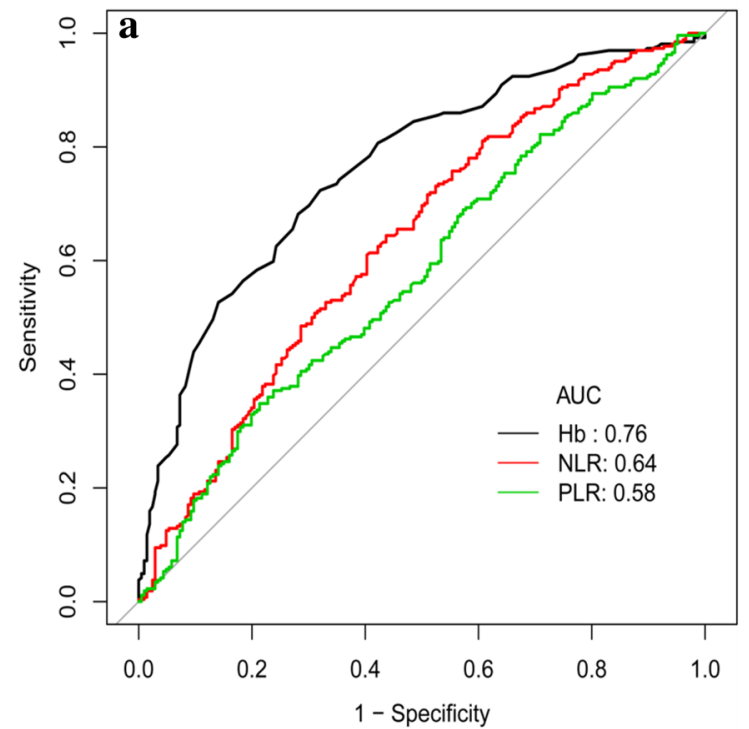

Compare 2 models

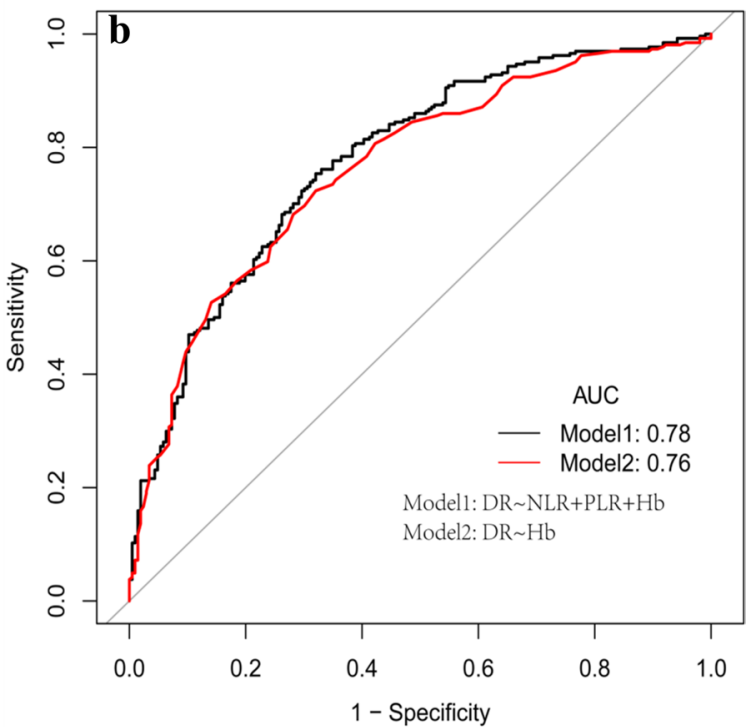

Fig. 2 Receiver operating characteristic (ROC) curves of NLR, PLR and Hb for DR a The ROC curves using Hb, NLR or PLR alone for discriminating DR ( $\mathrm{Hb}$, black, NLR, red, PLR, green) b The ROC curves included and not included NLR and PLR for predicting DR, (Model1: black, a combination of NLR, PLR and $\mathrm{Hb}$ for predicting DR, Model2: red, using $\mathrm{Hb}$ along to predict DR)

family history.(NRI(95\%CI) $0.53(0.36-0.71) \quad \mathrm{p}<0.001$; IDI $(95 \% \mathrm{CI}) 0.03(0.01-0.04) ; \mathrm{p}<0.001$.)

\section{Discussion}

Our research demonstrated the association of systemic inflammatory response index with diabetic retinopathy among type 2 diabetic patients without related family histories. First of all, we verified that levels of NLR and PLR but not MLR were higher in the DR group. Furthermore, according to our multivariate analysis, not only did NRL serve as an independent risk factor but also the highest quartile of both NLR and PLR added risk to DR. More importantly, addition of NLR and PLR to Hb-based model improved reclassification of DR. Through our study, we provide the simple and available blood-based index for DR, promoting the risk stratification of DR among type 2 diabetic patients without family history.

Although the association between blood inflammatory index and DR drew much attention previously [12, 20-22],our study further deciphered the association and the clinical application of these indicators in T2DM patients without associated family history. Herein, we investigate the association between the indicators and DR more comprehensively in a larger population $(n=470)$ and revealed that a combination of NLR, PLR and $\mathrm{Hb}$ displayed significantly improved discriminability and raised sensitivity compared with using $\mathrm{Hb}$ alone. Therefore, combining the three factors might be helpful in clinical practice to improve the identification of DR in T2DM patients without family history of diabetes and hypertension.

Chronic inflammation plays an essential role in the initiation and progression of type 2 diabetes and further accelerates the deterioration of micro-angiopathy and macrovascular disease in patients with diabetes [23]. Previous studies have evidenced that peripheral blood leukocytes and their subgroups are associated with macrovascular and microvascular complications among patients with type 2 diabetes [24]. Specifically, peripheral blood leukocytes include lymphocytes, basophils, neutrophils, eosinophils, and monocytes, each type of which holds a unique biological function in systemic inflammation. NLR and PLR are two indexes that represent the integration of two factors and are considered to be new markers of the systemic inflammatory response [14]. Increasing studies have confirmed their association with type 2 diabetes $[14,25]$. DR is a common micro-angiopathic complication in diabetes. More and more evidence indicates that inflammation plays an important part in the early and progressive stage of DR [26-28], through inducing the formation of new blood vessels and macular edema [26], damaging the glial crosstalk and causing neuronal loss [29]. In addition, studies have also found that many inflammatory cytokines (such as CRP, TNF- $\alpha$ and VEGF, etc.) increase in patients with DR [30]. And intervention and regulation targeted at the inflammatory 
response in patients with diabetic retinopathy [31] can prohibit the progression of diabetes and retinopathy.

As two major indexes related to systemic inflammatory, PLR and NLR have been proved to be associated with diabetes and its complications [12, 14]. Apart from genetic epigenetic factors (family history of diabetes and hypertension), our study showed that higher NLR levels significantly increased the risk of DR, which is consistent with the previous results. But compared with the former one, our research has a larger sample size with an enrollment of 470 patients and also excludes the inheritable family history. NLR represents peripheral blood neutrophils to lymphocytes ratio, which integrates different but complementary immune pathways in circulating blood. First of all, elevated NLR can be a manifestation of an increased number of neutrophils, which adhere to the endothelial cell, leading to vascular endothelial damage, and in turn causing extensive chronic inflammation [11, 32]. Hence the NLR might reveal the enhanced microvascular inflammation in DR patients. Secondly, lymphocytes serve as a major part of the body's immune response. They have the ability to control and regulate inflammatory responses, and a relatively higher proportion of CD4 T cells were proved to be anti-atherosclerotic [33]. And our study found a decrease in absolute count of lymphocytes among peripheral blood in patients with high-level NLR (NLR:2.36 \pm 1.16 in DR group versus $1.97 \pm 1.06$ in NDR group, $\mathrm{p}<0.001$; absolute count of lymphocytes: $(1.98 \pm 0.64) * 10^{9} / \mathrm{L}$ in DR group versus $(2.11 \pm 0.67) * 10^{9} / \mathrm{L}$ in NDR group, $\left.\mathrm{p}=0.031\right)$, which indicates the possibility of insufficient immunoregulation due to the fewer lymphocytes, In addition, this study also demonstrated that NLR levels were positively correlated with the duration of diabetes, BUN, and Scr, which is consistent with the previous results [34].

Previous studies have found that PLR is closely related to diabetes and can be used to assess the progress of the disease [35], predict and evaluate the diabetes-related lower limb vascular disease [15], atherosclerosis and diabetic foot ulcers [36]. However, Atak et al. didn't found an association between PLR and DR [35], which might be attributed to the small sample size. In our study, though the PLR as a continuous variate didn't show independent association with DR, the highest quartile of PLR indeed adds more than 2-fold risk to the presence of DR. It has been commonly admitted that platelets participate in thrombosis. Moreover, until now, increasing studies have proved that platelets played an important role in the immuno-inflammatory response. Specifically, platelets can release a variety of immune-regulating cytokines, chemokines, and other mediators, thus regulating the inflammation response in blood vessels in an autocrine or paracrine manner [37]. Meanwhile, it could also regulate neutrophils, endothelial cells, and lymph directly, allowing them to recruit towards injured tissue [38]. Similarly, based on the regulatory function of platelets, increased PLR might represent the relatively active inflammatory response of platelets among DR patients. Additionally, our study also indicates a positive correlation between PLR and diabetes courses and a negative correlation between PLR and BMI. Taken together, we provide the large-sample size-based evidence for the role of PLR in DR, and further mechanism studies are also needed in the future.

We also proved that in diabetic patients without a family history of diabetes and hypertension, $\mathrm{Hb}$ levels were still negatively associated with the risk of DR independent of established factors. Hemoglobin is a special protein transporting oxygen within red blood cells. And low hemoglobin levels might lead to tissue ischemia and hypoxia, which is one of the key mechanisms of DR occurrence [39]. Studies have found that hemoglobin levels were negatively related to endothelial function and lower hemoglobin levels directly resulted in organ damage [40]. In addition, the level of hemoglobin is an indicator of the anemia condition in our body. According to previous studies, it has been found that the anemia patients held a high level of vascular endothelial growth factor (VEGF) which is closely related to retinal neovascularization $[41,42]$. Some studies indicated that anemia may enhance oxidative stress [43], because the antioxidant capacity of red cells can be damaged due to anemia [44], which in turn promotes oxidative stress and accelerates presence of DR [45]. In particular, in this study, a combination of systemic inflammation indicators NLR, PLR, and Hb was shown to increase the predictability of DR and help DR reclassification.

The established relationships between some clinical factors and DR in our patient's group were in line with previous ones. Firstly, we found that blood lipid was a risk factor for DR, and the results of this study are consistent with previous one [46], indicating higher TG increased the risk of DR. In addition, in consistency with previous studies, diabetic nephropathy significantly increases the risk of DR with shared pathological mechanism $[47,48]$. We also observed higher levels of BUN and Scr in DR patients, revealing a potential interaction multi-organ complication under the background of diabetes.

In summary, in people with type 2 diabetes without a family history of diabetes and hypertension, systemic inflammation indicators NLR and PLR are closely related to DR. Higher NLR and PLR increase the risk of DR, and after combined with $\mathrm{Hb}$ indicators, they contributed to DR reclassification. To provide more practical and reliable guidance for clinical diagnosis, further multi-center prospective clinical studies and basic researches are also 
required to elucidate the relationship between the PLR NLR and DR.

\section{Limitations}

A series of limitations still exist in our study. First of all, our study is a single-center study, patients' recruitment, staff characteristics, and departmental protocols might add a limitation to the universality of our results. Additionally, with a lack of follow-up, we were unable to verify the predictive value of the factors, the further prospective cohort is still required to clarify the value PLR, NLR in predicting DR among T2DM patients in clinical practice. Finally, as our participants were type 2 diabetic patients without family history of hyperextension and diabetes, the results might not be extrapolated to other populations.

\section{Conclusion}

Systemic inflammatory response indexes NLR and PLR were associated with the presence of DR among patients without related family history and improved discriminability and re-classification of hemoglobin-based predictive model.

\section{Abbreviations}

DR: Diabetic retinopathy; DM: Diabetes mellitus; NLR: Neutrophil-to-lymphocyte ratio; PLR: Platelet-to-lymphocyte ratio; T2DM: Type 2 diabetes mellitus; NDR: Non-diabetic retinopathy; Hb: Hemoglobin; AUC: Area under curve; NRI: Net reclassification improvement; IDI: Integrated discrimination improvement; MLR: Monocyte-to-lymphocyte ratio; FPG: Fasting blood glucose; HbA1c: Glycosylated hemoglobin A1c; TG: Triglyceride; TC: Total cholesterol; LDL-C: Lowdensity lipoprotein cholesterol; HDL-C: High-density lipoprotein cholesterol; Scr: Serum creatinine; BUN: Blood urea nitrogen; SD: Standard deviation; WBC: White blood cell; AIC: Akaike information criterion; BMI: Body mass index.
\end{abstract}

\section{Acknowledgements}

Not applicable.

\section{Authors' contributions}

JRW and ZLC contributed equally to analysis and manuscript preparation; YY and $\mathrm{KY}$ was responsible for revising the manuscript for important intellectual content and final approval of the version of the article to be published; HJY, ZZJ, CFB, JMD and YCY were responsible for collecting data and helped perform the analysis with constructive discussions. KY, WYT, YPL, YYZ and XQG revised the manuscript; All authors read and approved the final manuscript.

\section{Funding}

This study was supported by the Endocrine Clinical Medical Center of Yunnan Province, No. ZX2019-02-02; the Natural Science Foundation of China (No. 81760734; No. 31660313); the Natural Science Foundation of Yunnan Province (No. 2017FA048; No. 2017FE467); the fund of the Diabetic Innovation Team (No. 2019HC002); and the fund for medical leaders in Yunnan Province (No. L-201609).

\section{Availability of data and materials}

The datasets used during the present study are available from the corresponding author on reasonable request.

\section{Ethics approval and consent to participate}

This study was approved by the ethics committee of the Fourth Affiliated Hospital of Kunming Medical University (No.2020039).
Consent for publication

Not applicable.

\section{Competing interests}

The authors declare that they have no competing interests.

\section{Author details}

${ }^{1}$ Department of Endocrinology and Metabolism, The Second People's Hospital of Yunnan Province, Fourth Affiliated Hospital of Kunming Medical University, Kunming 650021, China. ${ }^{2}$ Department of Cardiology, Ruijin Hospital, Shanghai Jiaotong University School of Medicine, 200001 Shanghai, China. ${ }^{3}$ The Second People's Hospital of Qujing City, Yunnan, Qujing 655000, China.

Received: 26 April 2020 Accepted: 24 June 2020

Published online: 02 July 2020

\section{References}

1. Solomon SD, Chew E, Duh EJ, Sobrin L, Sun JK, VanderBeek BL, et al. Diabetic retinopathy: a position statement by the American Diabetes Association. Diabet Care. 2017:40:412-8.

2. Chinese Medical Association Diabetes Division Retinopathy Group. Expert consensus on prevention and treatment of diabetic retinopathy. Chinese J Diabet Mellitus. 2018;10:241-7.

3. Song SJ, Wong TY. Current concepts in diabetic retinopathy. DiabetMetab J. 2014;38:416-25

4. Kuo JZ, Wong TY, Rotter Jl. Challenges in elucidating the genetics of diabetic retinopathy. JAMA Ophthalmol. 2014;132:96-107.

5. Wat N, Wong RL, Wong IY. Associations between diabetic retinopathy and systemic risk factors. Hong Kong Med J. 2016:22:589-99.

6. Molyneaux L, Constantino M, Yue D. Strong family history predicts a younger age of onset for subjects diagnosed with type 2 diabetes. Diabet Obes Metab. 2004;6:187-94

7. Yamamoto-Honda R, Takahashi Y, Mori Y, Yamashita S, Yoshida Y, Kawazu S, et al. A positive family history of hypertension might be associated with an accelerated onset of type 2 diabetes: results from the National Center Diabetes Database (NCDD-02). Endocr J. 2017;64:515-20.

8. Anwar SB, Asif N, Naqvi SAH, Malik S. Evaluation of multiple risk factors involved in the development of diabetic retinopathy. Pak J Med Sci. 2019;35:156-60.

9. Hu Y, Teng W, Liu L, Chen K, Liu L, Hua R, et al. Prevalence and risk factors of diabetes and diabetic retinopathy in Liaoning province, China:a population-based crosssectional study. PLoS One. 2015;10:e0121477.

10. Hu Z, Tan S, Chen S, Qin S, Chen H, Qin S, et al. Diagnostic value of hematological parameters platelet to lymphocyte ratio and hemoglobin to platelet ratio in patients with colon cancer. Clin Chim Acta. 2019;501:48-52.

11. Azab B, Zaher M, Weiserbs KF, Torbey E, Lacossiere K, Gaddam S, et al. Usefulness of neutrophil to lymphocyte ratio in predicting short- and long-term mortality after non-ST-elevation myocardial infarction. Am J Cardiol. 2010;106:470-6.

12. Liu J, Liu X, Li Y, Quan J, Wei S, An S, et al. The association of neutrophil to lymphocyte ratio, mean platelet volume, and platelet distribution width with diabetic retinopathy and nephropathy: a meta-analysis. Biosci Rep. 2018;38:72.

13. Ulu SM, Dogan M, Ahsen A, Altug A, Demir K, Acartürk G, et al. Neutrophil-to-lymphocyte ratio as a quick and reliable predictive marker to diagnose the severity of diabetic retinopathy. Diabet Technol Ther. 2013;15:942-7.

14. Mertoglu C, Gunay M. Neutrophil-Lymphocyte ratio and Platelet-Lymphocyte ratio as useful predictive markers of prediabetes and diabetes mellitus. Diabetes Metab Syndr. 2017;11:S127-31.

15. Liu N, Sheng J, Pan T, Wang Y. Neutrophil to lymphocyte ratio and platelet to lymphocyte ratio are associated with lower extremity vascular lesions in Chinese patients with type 2 diabetes. Clin Lab. 2019. https://doi. org/10.7754/clin.lab.2018.180804.

16. Lee MK, Han KD, Lee JH, Sohn SY, Jeong JS, Kim MK, et al. High hemoglobin levels are associated with decreased risk of diabetic retinopathy in Korean type 2 diabetes. Sci Rep. 2018;8:5538. 
17. Traveset A, Rubinat E, Ortega E, Alcubierre N, Vazquez B, Hernández M, et al. Lower hemoglobin concentration is associated with retinal ischemia and the severity of diabetic retinopathy in type 2 diabetes. J Diabet Res. 2016;2016:3674946

18. Alberti KG, Zimmet PZ. Definition, diagnosis and classification of diabetes mellitus and its complications. Diabet Med. 1998;15:539-53.

19. Wilkinson CP, Ferris FL, Klein RE, Lee PP, Agardh CD, Davis M, et al. Proposed international clinical diabetic retinopathy and diabetic macular edema disease severity scales. Ophthalmology. 2003;110:1677-82.

20. Luo WJ, Zhang WF. The relationship of blood cell-associated inflammatory indices and diabetic retinopathy: a Meta-analysis and systematic review. Int J Ophthalmol. 2019;12:312-23.

21. Demirtas L, Degirmenci H, Akbas EM, Ozcicek A, Timuroglu A, Gurel A, et al. Association of hematological indicies with diabetes, impaired glucose regulation and microvascular complications of diabetes. Int J Clin Exp Med. 2015;8:11420-7.

22. Yue S, Zhang J, Wu J, Teng W, Liu L, Chen L. Use of the monocyte-tolymphocyte ratio to predict diabetic retinopathy. Int J Environ Res Public Health. 2015;12:10009-19.

23. Fujita T, Hemmi S, Kajiwara M, Yabuki M, Fuke Y, Satomura A, et al. Complement-mediated chronic inflammation is associated with diabetic microvascular complication. Diabetes Metab Res Rev. 2013;29:220-6.

24. Tong PC, Lee KF, So WY, Ng MH, Chan WB, Lo MK, et al. White blood cell count is associated with macro- and microvascular complications in chinese patients with type 2 diabetes. Diabet Care. 2004;27:216-22.

25. Sefil F, Ulutas KT, Dokuyucu R, Sumbul AT, Yengil E, Yagiz AE, et al. Investigation of neutrophil lymphocyte ratio and blood glucose regulation in patients with type 2 diabetes mellitus. J Int Med Res. 2014;42:581-8.

26. Kern TS. Contributions of inflammatory processes to the development of the early stages of diabetic retinopathy. Exp Diabet Res. 2007;2007:95103.

27. Rübsam A, Parikh S, Fort PE. Role of Inflammation in diabetic retinopathy. Int J Mol Sci. 2018;19:942.

28. Tang J, Kern TS. Inflammation in diabetic retinopathy. Prog Retin Eye Res. 2011;30:343-58.

29. Lange C, Storkebaum E, de Almodovar CR, Dewerchin M, Carmeliet P. Vascular endothelial growth factor: A neurovascular target in neurological diseases. Nat Rev Neurol. 2016;12:439-54.

30. Nalini M, Raghavulu BV, Annapurna A, Avinash P, Chandi V, Swathi N. Correlation of various serum biomarkers with the severity of diabetic retinopathy. Diabetes Metab Syndr. 2017;11:S451-4.

31. Malukiewicz G, Stafiej J, Lesiewska H, Sikorski B. Use of nonsteroidal antiinflammatory drugs in diabetic retinopathy. Klinika oczna. 2016:118:44-7.

32. Sala A, Folco G. Neutrophils, endothelial cells, and cysteinyl leukotrienes: a new approach to neutrophil-dependent inflammation? Biochem Biophys Res Commun. 2001;283:1003-6.

33. Wang RT, Zhang JR, Li Y, Liu T, Yu KJ. Neutrophil-Lymphocyte ratio is associated with arterial stiffness in diabetic retinopathy in type 2 diabetes. J Diabet Compli. 2015;29:245-9.

34. Chittawar S, Dutta D, Qureshi Z, Surana V, Khandare S, Dubey TN. Neutrophil-lymphocyte ratio is a novel reliable predictor of nephropathy, retinopathy, and coronary artery disease in indians with type-2 diabetes. Indian J Endocrinol Metab. 2017;21:864-70.

35. Atak B, Aktas G, Duman TT, Erkus E, Kocak MZ, Savli H. Diabetes control could through platelet-to-lymphocyte ratio in hemograms. Rev Assoc Med Bras. 1992;2019(65):38-42.

36. Mineoka Y, Ishii M, Hashimoto Y, Yamashita A, Nakamura N, Fukui M. Platelet to lymphocyte ratio correlates with diabetic foot risk and foot ulcer in patients with type 2 diabetes. Endocr J. 2019;66:905-13.

37. Chatterjee M, Geisler T. Inflammatory Contribution of Platelets Revisited: new Players in the Arena of Inflammation. Semin Thromb Hemost. 2016:42:205-14.

38. Jenne CN, Urrutia R, Kubes P. Platelets: bridging hemostasis, inflammation, and immunity. Int J Lab Hematol. 2013;35:254-61.

39. Irace C, Scarinci F, Scorcia V, Bruzzichessi D, Fiorentino R, Randazzo G, et al. Association among low whole blood viscosity, haematocrit, haemoglobin and diabetic retinopathy in subjects with type 2 diabetes. $\mathrm{Br}$ J Ophthalmol. 2011;95:94-8.

40. Sonmez A, Yilmaz MI, Saglam M, Kilic S, Eyileten T, Uckaya G, et al. The relationship between hemoglobin levels and endothelial functions in diabetes mellitus. Clin J Am Soc Nephrol. 2010;5:45-50.

41. Dunst J, Becker A, Lautenschläger C, Markau S, Becker H, Fischer K, et al. Anemia and elevated systemic levels of vascular endothelial growth factor (VEGF). Strahlenther Onkol. 2002;178:436-41.

42. Aiello LP, Pierce EA, Foley ED, Takagi H, Chen H, Riddle L, et al. Suppression of retinal neovascularization in vivo by inhibition of vascular endothelial growth factor (VEGF) using soluble VEGF-receptor chimeric proteins. Proc Natl Acad Sci USA. 1995;92:10457-61.

43. Chung JO, Park SY, Chung DJ, Chung MY. Relationship between anemia serum bilirubin concentrations, and diabetic retinopathy in individuals with type 2 diabetes. Medicine. 2019;98:e17693.

44. Grune T, Sommerburg O, Siems WG. Oxidative stress in anemia. Clin Nephrol. 2000;53:S18-22.

45. Kowluru RA, Mishra M. Oxidative stress, mitochondrial damage and diabetic retinopathy. Biochim Biophys Acta. 2015;1852:2474-83.

46. Liu Y,Yang J,Tao L,LV H,Jiang X,Zhang M,et al.Risk factors of diabetic retinopathy and sight-threatening diabetic retinopathy: a cross-sectional study of 13473 patients with type 2 diabetes mellitus in mainland China. BMJ Open.2017; 7:e016280.

47. Pedro RA, Marc BB, Raul NG, Antonio MR, Aida VM, Ramon SA, et al. Glomerular filtration rate and/or ratio of urine albumin to creatinine as markers for diabetic retinopathy: a ten-year follow-up study. J Diabet Res. 2018:2018:1-9.

48. Phipps JA, Feener EP. The kallikrein-kinin system in diabetic retinopathy: lessons for the kidney. Kidney Int. 2008;73:1114-9.

\section{Publisher's Note}

Springer Nature remains neutral with regard to jurisdictional claims in published maps and institutional affiliations.

Ready to submit your research? Choose BMC and benefit from

- fast, convenient online submission

- thorough peer review by experienced researchers in your field

- rapid publication on acceptance

- support for research data, including large and complex data types

- gold Open Access which fosters wider collaboration and increased citations

- maximum visibility for your research: over $100 \mathrm{M}$ website views per year

At $\mathrm{BMC}$, research is always in progress.

Learn more biomedcentral.com/submissions 